\title{
COMMUNICATIONS
}

\section{Reaction of 2,3-Dioxopyrrolo[2,1-a]isoquinolines with Naphthalene-2,3-diamine}

\author{
O. V. Surikova, A. G. Mikhailovskii, and M. I. Vakhrin \\ Perm State Pharmacentical Academy, ul. Polevaya 2, Perm, 614990 Russia \\ e-mail:perm@pfa.ru
}

Received February 23, 2010

DOI: $10.1134 / \mathrm{S} 1070428011040269$

We previously studied reactions of 2,3-dioxopyrrolo[2,1- $a$ ] isoquinolines with $o$-phenylenediamine $[1,2]$. Reactions of these compounds with bulkier binucleophiles were not reported. While performing studies in this line, we found that dioxopyrrolo[2,1-a]isoquinoline I reacts with naphthalene-2,3-diamine on heating in boiling glacial acetic acid to produce fused hexacyclic system III. By reaction of naphthalene-2,3diamine with analogous tetracyclic compound II of the benzo[f]isoquinoline series we obtained heptacyclic structure IV. Compounds III and IV attract interest as new synthons, biologically active substances, and luminophors [3].

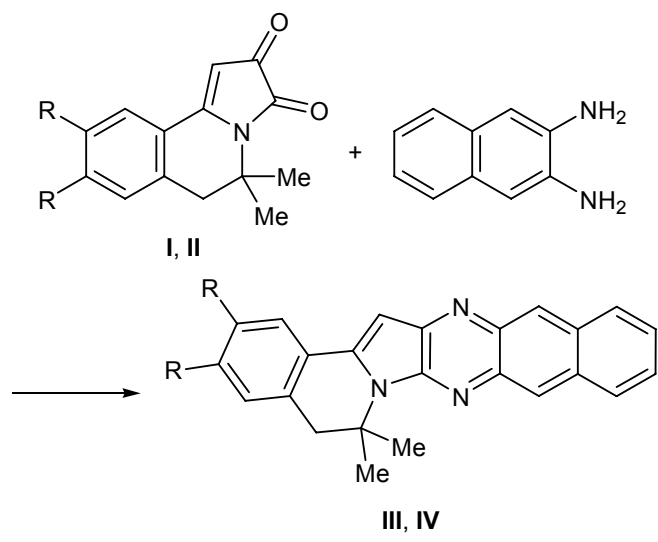

I, III, $\mathrm{R}=\mathrm{H}$; II, IV, $\mathrm{RR}=-\mathrm{CH}=\mathrm{CH}-\mathrm{CH}=\mathrm{CH}-$.

6,6-Dimethyl-5,6-dihydrobenzo $[g]$ benzo $[7,8]$ indolizino[2,3-b]quinoxaline (III). A mixture of $2.27 \mathrm{~g}$ (10 mmol) of compound $\mathbf{I}$ and $1.58 \mathrm{~g}(10 \mathrm{mmol})$ of naphthalene-2,3-diamine in $10 \mathrm{ml}$ of glacial acetic acid was heated for 30 min under reflux, the progress of the reaction being monitored by TLC. The mixture was then cooled to $20^{\circ} \mathrm{C}$ and diluted with $100 \mathrm{ml}$ of water, and the precipitate was filtered off, washed on a filter with $25 \%$ aqueous ammonia and water, dried, and recrystallized from isopropyl alcohol. Yield $2.31 \mathrm{~g}$ (66\%), yellow crystals, mp $222-224^{\circ} \mathrm{C} .{ }^{1} \mathrm{H}$ NMR spectrum, $\delta$, ppm: $1.92 \mathrm{~s}\left(6 \mathrm{H}, \mathrm{CH}_{3}\right), 3.13 \mathrm{~s}(2 \mathrm{H}, 6-\mathrm{H}), 7.00 \mathrm{~s}$ $(1 \mathrm{H}, 1-\mathrm{H}), 7.21-8.58 \mathrm{~m}\left(10 \mathrm{H}, \mathrm{H}_{\text {arom }}\right)$. Mass spectrum, $m / z\left(I_{\text {rel }}, \%\right): 349(67)[M]^{+}, 334(45)\left[M-\mathrm{CH}_{3}\right]^{+}, 319$ (55) $\left[M-2 \mathrm{CH}_{3}\right]^{+}$. Found, \%: C 82.43; H 5.41; $\mathrm{N}$ 12.12. $\mathrm{C}_{24} \mathrm{H}_{19} \mathrm{~N}_{3}$. Calculated, \%: C 82.49; H 5.48; N 12.03. M 349.43.

6,6-Dimethyl-5,6-dihydrobenzo $[g]$ naphtho$\left[1^{\prime}, 2^{\prime}: 7,8\right]$ indolizino $[2,3-b] q u i n o x a l i n e ~(I V)$ was synthesized in a similar way from $2.77 \mathrm{~g}(10 \mathrm{mmol})$ of compound II [4]. Yield 71\%, yellow crystals, mp 268$270^{\circ} \mathrm{C} .{ }^{1} \mathrm{H}$ NMR spectrum, $\delta$, ppm: $2.00 \mathrm{~s}\left(6 \mathrm{H}, \mathrm{CH}_{3}\right)$, $3.60 \mathrm{~s}(2 \mathrm{H}, 6-\mathrm{H}), 7.08 \mathrm{~s}(1 \mathrm{H}, 1-\mathrm{H}), 7.16-8.66 \mathrm{~m}(12 \mathrm{H}$, $\left.\mathrm{H}_{\text {arom }}\right)$. Mass spectrum, $m / z\left(I_{\text {rel }}, \%\right): 399(16)[M]^{+}, 384$ (8) $\left[M-\mathrm{CH}_{3}\right]^{+}, 369(20)\left[M-2 \mathrm{CH}_{3}\right]^{+}$. Found, \%: C 84.12; H 5.23; N 10.63. $\mathrm{C}_{28} \mathrm{H}_{21} \mathrm{~N}_{3}$. Calculated, \%: C 84.18; H 5.30; N 10.52. M 339.49.

The ${ }^{1} \mathrm{H}$ NMR spectra were recorded on a Bruker300 spectrometer $(300 \mathrm{MHz})$ from solutions in $\mathrm{CDCl}_{3}$ using HMDS as internal reference $(\delta 0.05 \mathrm{ppm})$. The mass spectra (electron impact, $70 \mathrm{eV}$ ) were obtained on a Finnigan MAT INCOS 50 instrument.

\section{REFERENCES}

1. Mikhailovskii, A.G., Shklyaev, V.S., and Aleksandrov, B.B., Khim. Geterotsikl. Soedin., 1990, p. 808.

2. Surikova, O.V., Aliev, Z.G., Polygalova, N.N., Mikhailovskii, A.G., and Vakhrin, M.I., Russ. J. Org. Chem., 2008, vol. 44, p. 901.

3. Mikhailovskii, A.G. and Shklyaev, V.S., Khim. Geterotsikl. Soedin., 1995, p. 650.

4. Aleksandrov, B.B., Shklyaev, V.S., and Shklyaev, Yu.V., Khim. Geterotsikl. Soedin., 1992, p. 375. 